\section{Nonmyeloablative, HLA-haploidentical bone marrow transplantation with high dose, post-transplantation cyclophosphamide}

Ashley Munchel, 1 Chimen Kesserwan, 1

Heather J. Symons, ${ }^{1}$ Leo Luznik,²

Yvette L. Kasamon, ${ }^{2}$ Richard J. Jones, ${ }^{2}$

Ephraim J. Fuchs²

1Divisions of Pediatric Oncology, Sidney Kimmel Comprehensive Cancer Center at Johns Hopkins, Baltimore, Maryland, USA; ${ }^{2}$ Divisions of Hematologic Malignancies, Sidney Kimmel

Comprehensive Cancer Center at Johns Hopkins, Baltimore, MD, USA

\section{Abstract}

Allogeneic stem cell transplantation (SCT) from an HLA-haploidentical relative provides a potentially curative treatment option for hematologic malignancies patients who lack a suitably HLA-matched donor. The greatest challenge to performing HLA-haploidentical SCT has been high rates of graft failure and severe graft-versus-host disease (GVHD). Our group has been exploring high dose, post-transplantation cyclophosphamide (Cy) as prophylaxis of GVHD after nonmyeloablative, HLA-haploidentical bone marrow transplantation, or mini-haploBMT. Among 210 recipients of mini-haploBMT, $87 \%$ of patients have experienced sustained donor cell engraftment. The cumulative incidences of grades II-IV acute GVHD and chronic GVHD are $27 \%$ and $13 \%$, respectively. Five-year cumulative incidence of non-relapse mortality is $18 \%$, relapse is $55 \%$, and actuarial overall survival and event-free survivals are $35 \%$ and $27 \%$, respectively. These outcomes suggest that mini-haploBMT with post-transplantation Cy is associated with acceptably low toxicities and can provide longterm survival, if not cure, for many patients with advanced hematologic malignancies.

\section{Introduction}

\section{Post-transplantation Cy as a form of drug-induced immunologic tolerance}

The idea of giving post-transplantation $\mathrm{Cy}$ came from studies that have shown that immunologic tolerance can be induced by the sequence of administering an antigen followed shortly by giving a drug to kill proliferating, antigen-reactive $\mathrm{T}$ or B cells. This phenomenon of drug-induced immunological tolerance is not a new concept. It was initially reported in 1959 when it was shown that giving rabbits 6mercaptopurine prevented their ability to produce antibodies against human serum albumin. 1

Through a progression of studies it was found that $\mathrm{Cy}$ given one to three days after antigenic stimulation induces tolerance to tumor, skin grafts or other solid organ transplants in murine models. ${ }^{2-4}$ The proposed mechanism has been clonal destruction of alloreactive T cells. ${ }^{5}$ In other words, antigenic stimulation from the graft causes rapid proliferation of T-cell clones specific to the foreign antigen. When Cy is given at the height of proliferation, 1-3 days after stimulation, it inhibits DNA replication and results in selective destruction of alloreactive T-cell clones. In vitro studies have shown this occurs in mature $\mathrm{T}$ cells only. ${ }^{6}$ Importantly, Cy may not kill those quiescent $\mathrm{T}$ cells that were not activated by administration of the antigen. These cells include pathogen-specific memory $\mathrm{T}$ cells, and this explains why immunity to infection is spared among patients treated with high dose Cy outside the context of allogeneic SCT. Thus, the tolerance induced by post-transplantation $\mathrm{Cy}$ is specific to the administered antigen. When the antigen administered is allogeneic cells, post-transplantation Cy induces selective allodepletion.

Phase II trial of nonmyeloablative, HLA-haploidentical BMT with high-dose post-transplantation cyclophsphamide

Based upon encouraging results from a phase I/II trial,7,8 we recently completed a phase II trial of nonmyeloablative, HLA-haploidentical BMT with high-dose, post-transplantation Cy for patients with advanced hematologic malignancies. As of September 14th, 2010, 210 consecutive patients received nonmyeloablative, HLA-haploidentical transplantation with high-dose, post-transplantation cyclophosphamide and had at least one year of follow-up. Details concerning criteria for eligibility, HLA typing, outcomes measures, and statistical analysis have been published previously. 8,9

The treatment regimen given to all patients is shown in Figure 1. All patients were intended to be treated as outpatients. Conditioning comprised cyclophosphamide 14.5 $\mathrm{mg} / \mathrm{kg} /$ day on days -6 and -5 , fludarabine 30 $\mathrm{mg} / \mathrm{m}^{2} /$ day for five consecutive days starting on day -6 , and 2 Gy total body irradiation given in a single fraction on day -1 . Bone marrow was harvested from donors and infused into recipients on day 0 . There was no manipulation to deplete graft T-cells. GVHD prophylaxis con-
Correspondence: Ephraim Joseph Fuchs, 288 Cancer Research Building I, 1650 Orleans Street, Baltimore, Maryland, USA 21231.

Tel: (410) 955-8143 - Fax: (410) 614-3809.

E-mail: fuchsep@jhmi.edu

Key words: .HLA-haploidentical bone marrow transplantation

Conflict of interest: The authors declare no conflicts of interest.

Authors contributions: AM, manuscript writing; CK, manuscript editing; HS, YK, RJ, research desing, patients management, data analysis, manuscript editing; LL, EF, research design, data analysis, manuscript writing.

Received for publication: 4 May 2011. Accepted for publication: 4 June 2011.

This work is licensed under a Creative Commons Attribution NonCommercial 3.0 License (CC BYNC 3.0).

(C) Copyright A. Munchel et al., 2011

Licensee PAGEPress, Italy

Pediatric Reports 2011; 3(s2):e15

doi:10.4081/pr.2011.s2.e15

sisted of cyclophosphamide $50 \mathrm{mg} / \mathrm{kg}$ IV, together with Mesna, each on days 3 and 4 , mycophenolate mofetil $15 \mathrm{mg} / \mathrm{kg}$ po tid (maximum $3 \mathrm{~g} /$ day) from day 5-35, and tacrolimus from day 5-180. Tacrolimus levels were monitored at least weekly with a desired concentration from 5-15 ng/ml. Prophylactic antimicrobial therapy was started on day -6 and included norfloxacin, fluconazole, appropriate prophylaxis of Pneumocystis carinii pneumonia, and valacyclovir.

Characteristics of the patients, donors, and grafts are shown in Table 1. All patients had poor risk hematologic malignancies that were judged to be incurable by chemotherapy alone. Fifty-eight patients had experienced relapse of their original malignancy after a prior autologous transplantation procedure, including 22 of the 30 patients with Hodgkin lymphoma and 23 of the 66 patients with non-Hodgkin lymphoma. Donors were selected primarily on the basis of health status, lack of anti-donor HLA antibodies in the recipient, and $\mathrm{ABO}$ and $\mathrm{CMV}$ compatibility, and without regard for the degree of HLA mismatch between donor and recipient.

\section{Engraftment and donor chimerism}

Of the 210 patients transplanted, 204 were evaluable for donor cell engraftment. Twentyseven patients (13\%) failed to engraft. Nearly all patients with primary or secondary graft 


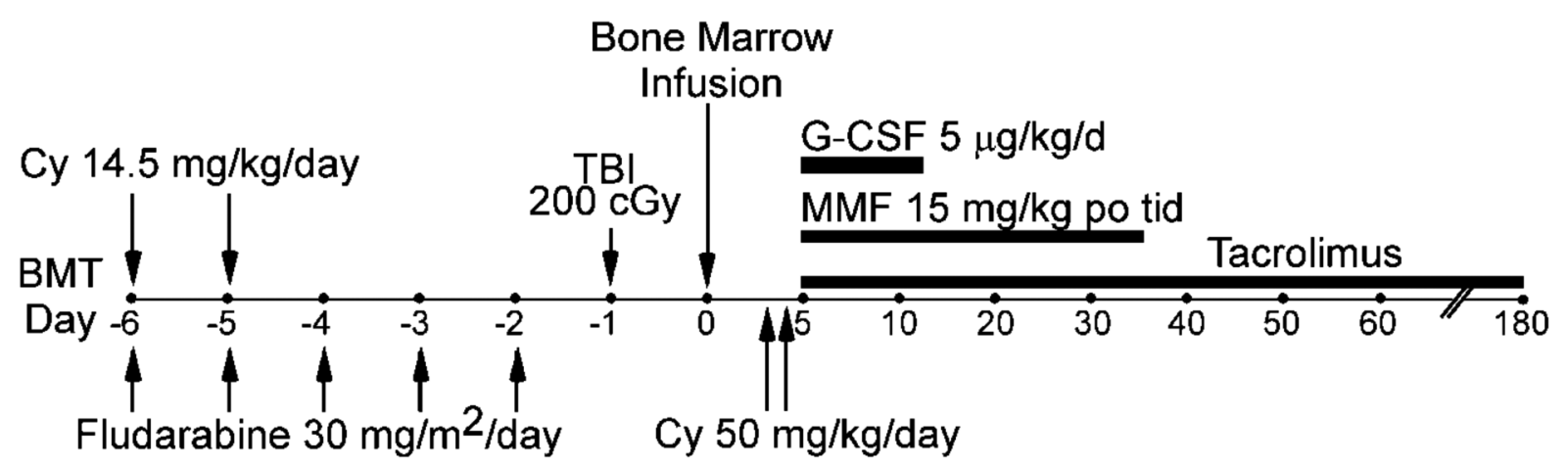

Figure 1. Treatment schema for nonmyeloablative conditioning regimine in HLA-haploidentical transplantation with post-transplantation cyclophosphamide. $\mathrm{MMF}=$ mycophenolate mofetil; $\mathrm{TBI}=$ total body irradiation; $\mathrm{Cy}=$ cyclophosphamide; $\mathrm{G}-\mathrm{CSF}=$ granulocyte colony stimulating factor.

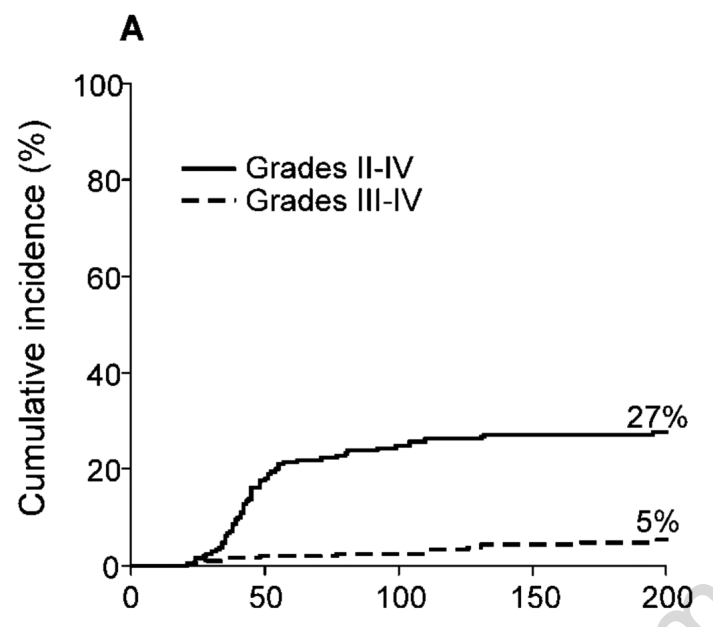

Days after transplantation

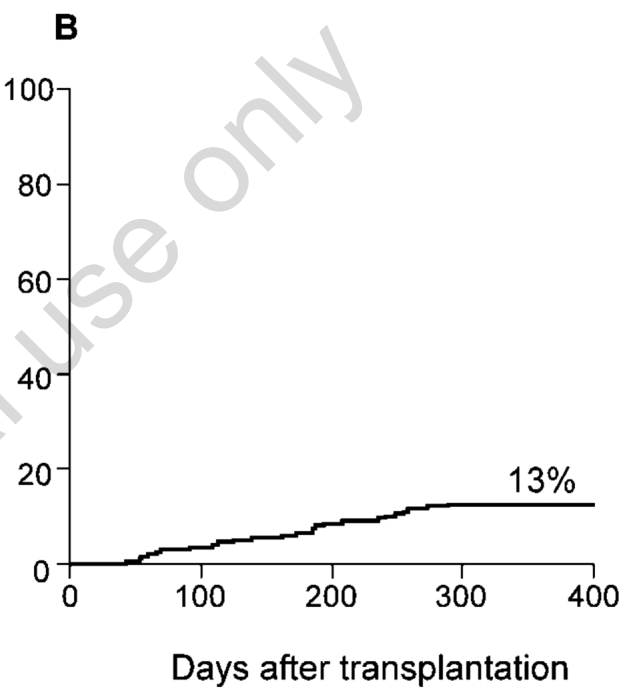

Figure 2. Cumulative incidence of acute (A) and chronic (B) GVHD after nonmyeloablative haploidentical stem cell transplantation with post-transplantation cyclophosphamide. $n=210$

Table 1. Patient, donor, and graft characteristics.

\begin{tabular}{lc}
\hline Patient age (median, range) & $52(1-73)$ \\
Sex (male/female) & $149 / 61$ \\
\hline Diagnoses: & 43 \\
Acute myeloid leukemia & 16 \\
Acute lymphocytic leukemia & 9 \\
Chronic myeloid leukemia & 24 \\
Chronic lymphocytic leukemia & 11 \\
Myelodysplastic syndrome & 30 \\
Hodgkin lymphoma & 66 \\
Non-Hodgkin lymphoma & 6 \\
Multiple myeloma & 5 \\
Myeloproliferative disorder & $42(14-73)$ \\
Donor age (median, range) & 35 \\
Parents & 102 \\
Siblings or half-siblings & 73 \\
Children & 3.7 \\
\hline Graft nucleated cell dose (x 108/kg) & 3.7 \\
T cell dose (x 107/kg) & 3.7 \\
\hline CD34+ cell dose (x 106/kg) & $4 / 10^{*}$ \\
Median donor/recipient HLA antigen mismatch & \\
\hline
\end{tabular}

*Patients and donors were typed at high resolution for HLA-A, -B, -Cw, -DRB1, and -DQB1. failure experienced recovery of autologous hematopoiesis. As reported previously, ${ }^{8}$ the median time to a neutrophil count of $\geq 500 / \mu \mathrm{L}$ was 15 days, and the median time to an unsupported platelet count of $\geq 20,000 / \mu \mathrm{L}$ was 24 days.

\section{GVHD}

Figure 2 shows that the cumulative incidence of grade 2-4 aGVHD was $27 \%$, grade $3-4$ aGVHD was 5\% and chronic GVHD was $13 \%$. This coincides with the data previously reported in the 67 patients, which had shown a cumulative incidence of grade 2-4 aGVHD of $34 \%$, grade $3-4$ aGVHD of $6 \% .8$

\section{Relapse and nonrelapse mortality}

The cumulative incidences of relapse and nonrelapse mortality were $55 \%$ and $18 \%$, respectively (Figure 3). One hundred thirteen 


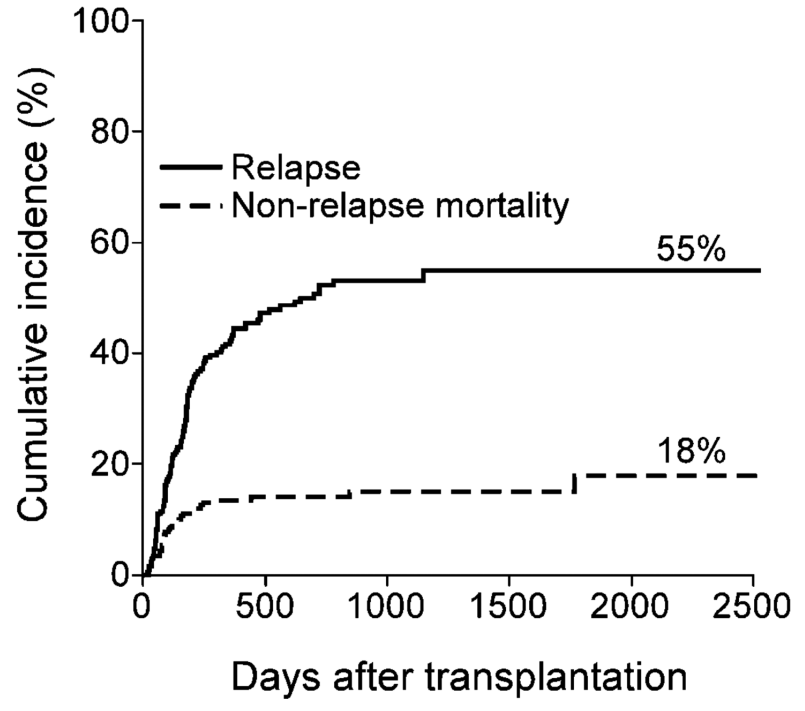

Figure 3. Cumulative incidence of relapse and nonrelapse mortality after nonmyeloablative haploidentical stem cell transplantation with post-transplantation cyclophosphamide.

patients have died. The causes of death were relapse $(n=79)$, infection $(n=15)$, pulmonary complications $(n=7)$, GVHD $(n=5)$, other $(\mathrm{n}=4)$, or unknown $(\mathrm{n}=3)$.

\section{Overall and event-free survival}

Three-year overall survival and event free survival are $41 \%$ and $32 \%$ respectively (Figure 4). Three year overall survival was
$50 \%$ for patients transplanted for acute lymphocytic leukemia, $45 \%$ for patients transplanted for myelodysplastic syndrome or myeloproliferative disorder, and $35 \%$ for patients transplanted for acute myeloid leukemia (Figure 4B). Three year survival was $62 \%$ for 30 patients with Hodgkin lymphoma (22 of whom had undergone prior autologous SCT), $41 \%$ for patients with nonHodgkin lymphoma, and only $22 \%$ for patients with chronic lymphocytic leukemia (Figure 4C).

\section{Effect of HLA-disparity} on outcome of HLA-haploidentical SCT with post-transplantation cyclophosphamide

Previous studies of T cell-replete, HLA-haploidentical BMT after myeloablative conditioning have shown that increasing HLA mismatch between donor and recipient was associated with worse survival due to an increased incidence of GVHD and NRM, which outweighed any potential reduction in the incidence of relapse. ${ }^{10-12}$ Since our regimen incorporated nonmyeloablative conditioning and novel GVHD prophylaxis, we examined the impact of increasing HLA disparity on outcome. ${ }^{9}$
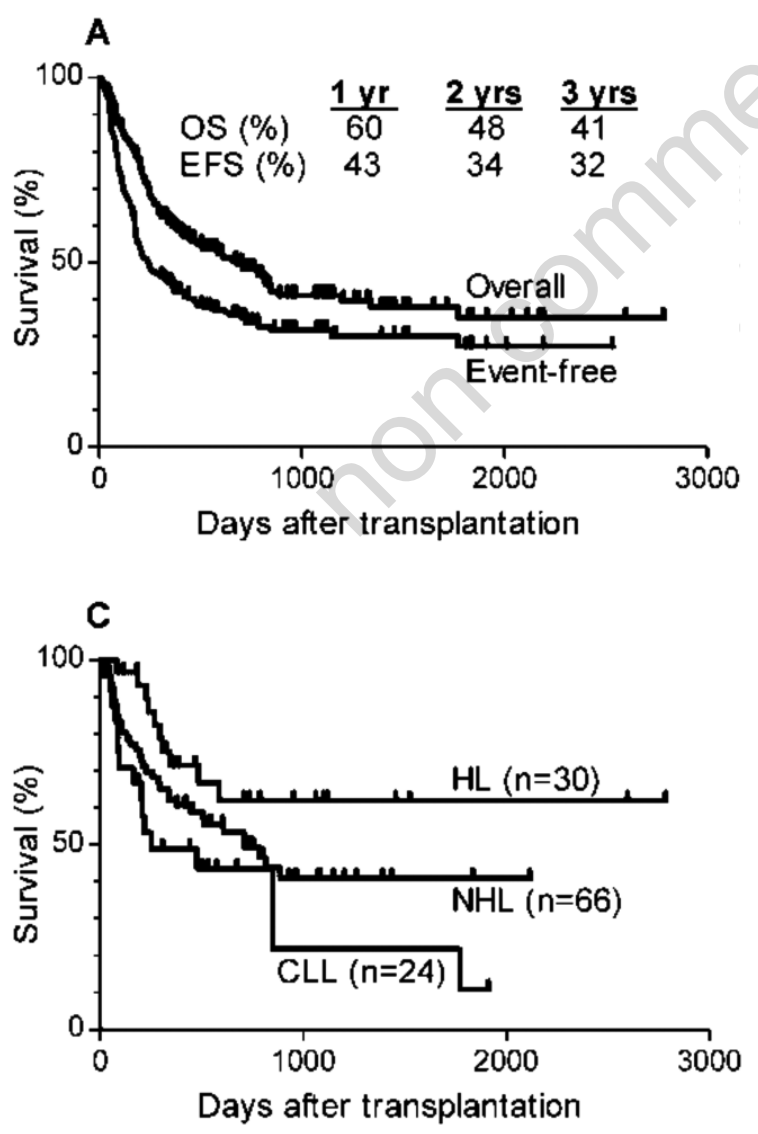

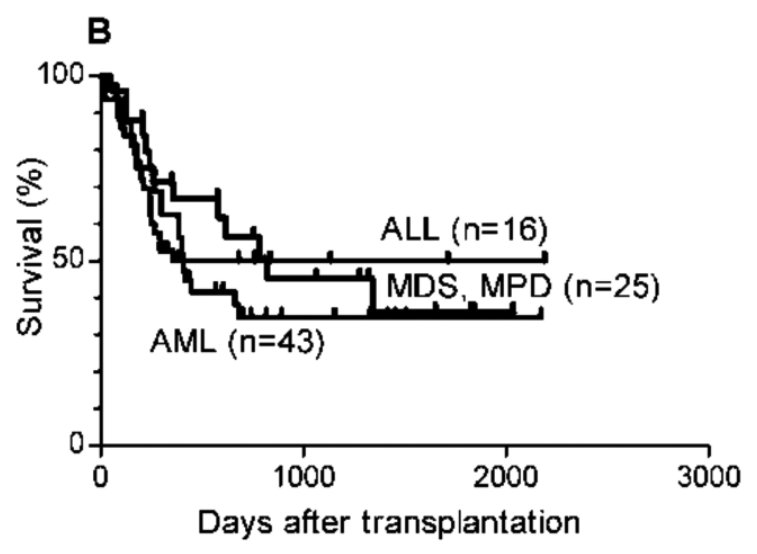

Figure 4. Actuarial curves of (A) overall survival (OS) and event-free survival (EFS) in all patients undergoing nonmyeloablative haploidentical stem cell transplantation with post-transplantation cyclophosphamide; (B) overall survival in patients with acute lymphocytic leukemia (ALL), acute myelocytic leukemia (AML) or myelodysplastic syndrome (MDS) or myeloproliferative disorder (MPD); (C) overall survival in patients with Hodgkin lymphoma (HL), non-Hodgkin lymphoma (NHL) and chronic lymphocytic lymphoma (CLL). 
gene for the CCR5 receptor for the human im-

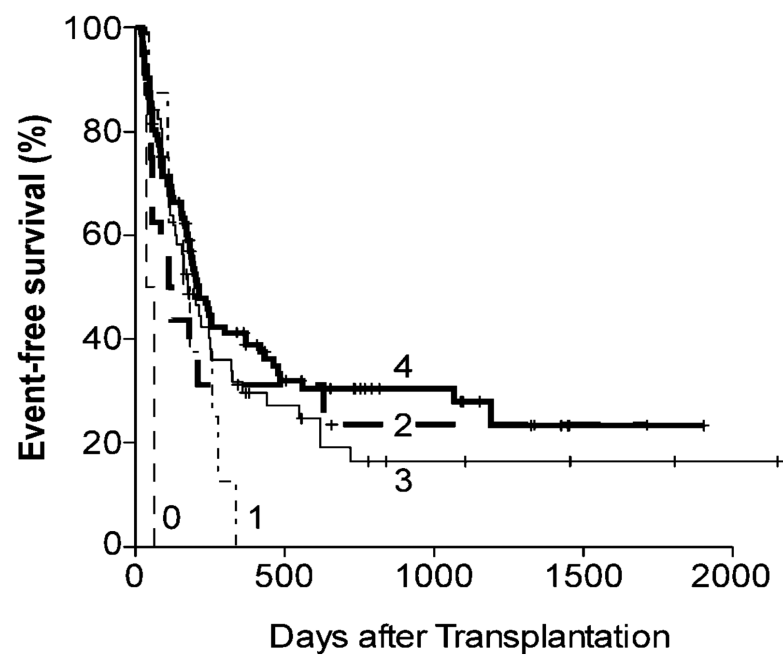

Figure 5. Event-free survival (EFS) of patients undergoing nonmyeloablative haploidentical stem cell transplantation with post-transplantation cyclophosphamide according to number of nismatched HLA-antigens in any direction (GVH or HVG).

Interestingly, Figure 5 shows a trend to improved event-free survival with increasing HLA antigen disparity between donor and recipient $(\mathrm{HR}=0.80 ; 95 \%$ confidence interval $[\mathrm{CI}]=$ $0.66-0.96 ; \mathrm{P}=.02$ ), with the hazard ratio of 0.8 indicating a $20 \%$ reduction in the risk of an event (death or relapse) for each increment of HLA mismatch. An HLA-DRB1 antigen mismatch in the graft-versus-host direction, and two or more HLA Class I (HLA-A, -B, and -Cw) mismatches in either direction were found to be associated with decreased incidences of relapse without an increased incidence of nonrelapse mortality (data not shown). While an analysis of the effects of NK cell alloreactivity on outcome is ongoing, we have previously found that donor-recipient mismatches at genes for inhibitory Killer Immunoglobulinlike Receptors, or iKIRs, or KIR haplotypes, were associated with improved outcome of nonmyeloablative, HLA-haploidentical BMT with post-transplantation cyclophosphamide. ${ }^{13}$

\section{Discussion}

The major advantage of HLA-haploidentical stem cell transplantation is rapid and near universal availability of one or more donors, since all biological parents and children, and $50 \%$ of siblings or half-siblings of a patient are HLAhaploidentical. Indeed, in our clinical trial the patients have had an average of four potential HLA-haploidentical donors for transplantation. The major disadvantages of this approach have been the consequences of intense bi-directional alloreactivity, i.e. high incidences of graft failure, severe GVHD, non-relapse mortality, and severe opportunistic infections re- sulting from poor immune reconstitution. High-dose, post-transplantation Cy was developed in mouse models of solid organ and hematopoietic stem cell transplantation as a method of achieving selective depletion of alloreactive T cells in vivo.2,3,14-17 The studies described here show that high dose, post-transplantation Cy enables HLA-haploidentical stem cell transplantation after nonmyeloablative conditioning with acceptably low incidences of fatal graft rejection, severe GVHD, and non-relapse mortality. The low death rate from opportunistic infection is testimony to effective clinical immune reconstitution after transplantation. Interestingly, post-transplantation Cy appears to nullify the detrimental impact of HLA mismatching on the outcome of allogeneic SCT for hematologic malignancies. ${ }^{9}$ The practical consequence of this finding is that stem cell donors may be selected without regard to the degree of donor-recipient HLA mismatching, and on the basis of biologic or genetic characteristics other than HLA genes. For example, we are currently conducting a clinical trial of nonmyeloablative allogeneic SCT and post-transplantation rituximab therapy of lymphoma, in which donors are selected according to Fc(gamma) RIIIa (CD16) genetic polymorphisms that predict clinical responsiveness to rituximab therapy. 18,19 HLA-haploidentical SCT expands access to transplantation for patients with sickle cell disease, who have an $18 \%$ chance of having a disease-free, HLA-matched sibling. ${ }^{20}$ We are currently testing whether post-transplantation $\mathrm{Cy}$ permits HLA mismatching in unrelated donor transplantation. If so, it may be possible to select unrelated donors who are partially HLA mismatched but who harbor a mutation in the munodeficiency virus. Such individuals are genetically resistant to HIV infection, and as donors could provide a cure for AIDS through allogeneic stem cell transplantation. ${ }^{21,22}$ These examples provide a taste of the therapeutic possibilities enabled by a successful crossing of the HLA barrier. We are currently characterizing phenotypic and functional immune reconstitution after allogeneic SCT with posttransplantation $\mathrm{Cy}$, and the mechanisms of $\mathrm{Cy}$ induced tolerance in the human. With improvements in our understanding of Cy-induced tolerance, 23 and with continued efforts to improve the safety and efficacy of HLA-haploidentical SCT, we hope to offer this approach to cure increasing numbers of children and adults with hematologic malignancies, ${ }^{24}$ and extend this treatment to patients with lifethreatening, non-malignant hematologic disorders.

\section{References}

1. Schwartz R, Dameshek W. Drug-induced immunological tolerance. Nature 1959; 183:1682-3.

2. Berenbaum MC, Brown IN. Prolongation of homograft survival in mice with single doses of cyclophosphamide. Nature 1963; 200:84.

3. Nirmul G, Severin C, Taub RN. Cyclophosphamide-induced immunologic tolerance to skin homografts. Surg Forum 1971;22:287-8.

4. Mayumi H, Himeno K, Shin T, Nomoto K. Drug-induced tolerance to allografts in mice. VI. Tolerance induction in H-2-haplotype-identical strain combinations in mice. Transplantation 1985;40:188-94.

5. Eto M, Mayumi H, Tomita Y, et al. Sequential mechanisms of cyclophosphamide-induced skin allograft tolerance including the intrathymic clonal deletion followed by late breakdown of the clonal deletion. J Immunol 1990;145:1303-10.

6. Strauss G, Osen W, Debatin KM. Induction of apoptosis and modulation of activation and effector function in T cells by immunosuppressive drugs. Clin Exp Immunol 2002;128:255-66.

7. O'Donnell PV, Luznik L, Jones RJ, et al. Nonmyeloablative bone marrow transplantation from partially HLA-mismatched related donors using posttransplantation cyclophosphamide. Biol Blood Marrow Transplant 2002;8:377-386.

8. Luznik L, O'Donnell PV, Symons HJ, et al. HLA-Haploidentical Bone Marrow Transplantation for Hematologic Malignancies Using Nonmyeloablative Conditioning and High-Dose, Posttransplantation Cyclophosphamide. Biol Blood Marrow Transplant 


\section{8;14:641-50.}

9. Kasamon YL, Luznik L, Leffell MS, et al. Nonmyeloablative HLA-Haploidentical Bone Marrow Transplantation with High-Dose Posttransplantation Cyclophosphamide: Effect of HLA Disparity on Outcome. Biol. Blood Marrow Transplant 2010;16:482-9.

10. Anasetti C, Beatty PG, Storb R, et al. Effect of HLA incompatibility on graft-versushost disease, relapse, and survival after marrow transplantation for patients with leukemia or lymphoma. Hum Immunol 1990;29:79-91.

11. Szydlo R, Goldman JM, Klein JP, et al. Results of allogeneic bone marrow transplants for leukemia using donors other than HLA-identical siblings. J Clin Oncol 1997;15:1767-77.

12. Ash RC, Horowitz MM, Gale RP, et al. Bone marrow transplantation from related donors other than HLA- identical siblings: effect of $\mathrm{T}$ cell depletion. Bone Marrow Transplant 1991;7:443-52.

13. Symons HJ, Leffell MS, Rossiter ND, et al. Improved Survival with Inhibitory Killer Immunoglobulin Receptor (KIR) Gene Mismatches and KIR Haplotype B Donors after Nonmyeloablative, HLA-Haploiden- tical Bone Marrow Transplantation. Biol Blood Marrow Transplant 2010;16:533-42.

14. Mayumi H, Good RA. Long-lasting skin allograft tolerance in adult mice induced across fully allogeneic (multimajor $\mathrm{H}-2$ plus multiminor histocompatibility) antigen barriers by a tolerance-inducing method using cyclophosphamide. J Exp Med 1989;169:213-38.

15. Mayumi H, Umesue M, Nomoto K. Cyclophosphamide-induced immunological tolerance: an overview. Immunobiology 1996;195:129-39.

16. Colson YL, Li H, Boggs SS, et al. Durable mixed allogeneic chimerism and tolerance by a nonlethal radiation-based cytoreductive approach. J Immunol 1996;157:2820-9.

17. Luznik L, Jalla S, Engstrom LW, Iannone R, Fuchs EJ. Durable engraftment of major histocompatibility complex-incompatible cells after nonmyeloablative conditioning with fludarabine, low-dose total body irradiation, and posttransplantation cyclophosphamide. Blood 2001;98:3456-64.

18. Cartron G, Dacheux L, Salles G, et al. Therapeutic activity of humanized anti-CD20 monoclonal antibody and polymorphism in IgG Fc receptor FcgammaRIIIa gene. Blood
2002;99:754-8.

19. Weng WK, Levy R. Two immunoglobulin G fragment $C$ receptor polymorphisms independently predict response to rituximab in patients with follicular lymphoma. J Clin Oncol 2003;21:3940-7.

20. Mentzer WC, Heller S, Pearle PR, et al. Availability of related donors for bone marrow transplantation in sickle cell anemia. Am J Pediatr Hematol Oncol 1994;16:27-9.

21. Hutter G, Nowak D, Mossner M, et al. Long-Term Control of HIV by CCR5 Delta32/Delta32 Stem-Cell Transplantation. N Engl J Med 2009;360:692-8.

22. Allers K, Hutter G, Hofmann J, et al. Evidence for the cure of HIV infection by CCR5\{Delta\}32/\{Delta\}32 stem cell transplantation. Blood 2011;117:2791-9.

23. Luznik L, Fuchs E. High-dose, post-transplantation cyclophosphamide to promote graft- host tolerance after allogeneic hematopoietic stem cell transplantation. Immunol Res 2010;47:65-77.

24. Luznik L, Jones RJ, Fuchs EJ. High-dose cyclophosphamide for graft-versus-host disease prevention. Curr Opin Hematol 2010;17:493-9. 